\title{
How dispersal capacity makes non-dispersal functional traits of animal communities diverge or converge depending on spatial scale
}

\author{
Ning Ding ${ }^{1}$, Hans Cornelissen ${ }^{2}$, Beixin Wang ${ }^{1}$, and Matty Berg ${ }^{3}$ \\ ${ }^{1}$ Nanjing Agricultural University \\ ${ }^{2} \mathrm{VU}$ University Amsterdam \\ ${ }^{3}$ Affiliation not available
}

November 9, 2020

\begin{abstract}
Community assembly theory proposed a hierarchy by which local composition is determined by both regional processes, e.g. dispersal and migration, and local processes, e.g. local filtering by environmental conditions and competition. How these factors interact and combined govern local species assembly and trait composition is poorly known. Thereto, we propose a conceptual model in which strong dispersal ability (linked to low environmental tolerance) is fitness-dependent, promoting species to track environmental changes; weak dispersal (linked to high tolerance) should be fitness-independent, enhancing the influence of competition. Conquently, local trait dissimilarity should have a positive humpback shape, while regional trait dissimilarity should have an upturned-humpback relationship with a dispersal trait gradient. We found strong empirical support for this concept in trait distribution patterns of stream macroinvertebrates from five basins across China. These findings are discussed in terms of metacommunity theory and merit further testing of our concept across ecosystems and organism worldwide.
\end{abstract}

\section{Hosted file}

Main documents.pdf available at https://authorea.com/users/374146/articles/491743-howdispersal-capacity-makes-non-dispersal-functional-traits-of-animal-communities-divergeor-converge-depending-on-spatial-scale 